\title{
Luminous infrared starbursts in a cluster of galaxies
}

\author{
Pierre-Alain Duc ${ }^{1}$, Dario Fadda ${ }^{2}$, Bianca Poggianti ${ }^{3}$, David Elbaz ${ }^{1}$, \\ Andrea Biviano ${ }^{4}$, Hector Flores ${ }^{5}$, Alan Moorwood ${ }^{6}$, \\ Alberto Franceschini ${ }^{7}$ and Catherine Cesarsky ${ }^{6}$ \\ ${ }^{1}$ CEA-Saclay and CNRS FRE 2591, France, email: paduc@cea.fr \\ ${ }^{2}$ Caltech, SIRTF Science Centre, Pasadena, USA \\ ${ }^{3}$ Osservatorio Astronomico di Padova, Padova, Italy \\ ${ }^{4}$ INAF/Osservatorio Astronomico di Trieste, Trieste, Italy \\ ${ }^{5}$ Observatoire de Paris, GEPI, Meudon, France \\ ${ }^{6}$ European Southern Observatory, Garching, Germany \\ ${ }^{7}$ Dipartimento di Astronomia, Padova, Italy
}

\begin{abstract}
Analysing mid-infrared ISOCAM images of the cluster of galaxies J1888.16CL, we identified among its members several particularly active galaxies with total infrared luminosities well above $10^{11} \mathrm{~L}_{\odot}$. If powered by dust enshrouded starbursts, as suggested by their optical spectra, these Luminous Infrared Galaxies would exhibit star formation rates surprisingly high in the cluster environment. The triggering mechanism is unclear but could be tidal collisions within sub-structures or infalling groups.
\end{abstract}

\section{Introduction}

In the Universe, the galaxies forming stars with the highest rates and/or exhibiting the most extreme nuclear radiation turn out to be the most obscured ones. Their internal activity being partly dust-enshrouded, it remains underestimated in visible observing bands and shows up in the infrared regime. Such so-called Luminous Infrared Galaxies (LIRGs) seem to be more prevalent in relatively dense environments like groups where tidal galaxy interactions play a role in enhancing their activity. On the other hand, the densest regions like clusters are usually considered as being particularly unfavorable for the global activity of their host galaxies. The combined action of multiple high-speed collisions, of the global cluster tidal field, and of the ram-pressure exerted by the rich intra-cluster medium contribute to strip galaxies of their disk and halo gas reservoirs. Without this fuel, star formation is quenched and eventually stops while the galaxy center is no longer fed and any nuclear activity ends.

We present here data obtained with the ISOCAM camera on board the Infrared Space Observatory (ISO) that reveals the presence of confirmed cluster members with high infrared luminosities and inferred star formation rates of several tens of solar masses per year. These LIRGs were discovered in the X-ray luminous cluster J1888.16CL, situated at a redshift of 0.56 . The observations performed in the LW3 band centered at $15 \mu \mathrm{m}$ covered a strip of $3 \times 15$ square arcminutes. Follow-up ground based observations were carried out using ESO facilities in Chile. They consist of optical B,V,R WFI/2.2m images, near-infrared J,H,Ks SOFI/NTT images and optical FORS1/VLT multi-slit spectra. This collection of data allowed us to assess the cluster membership of the ISO sources and study their spectral properties. 


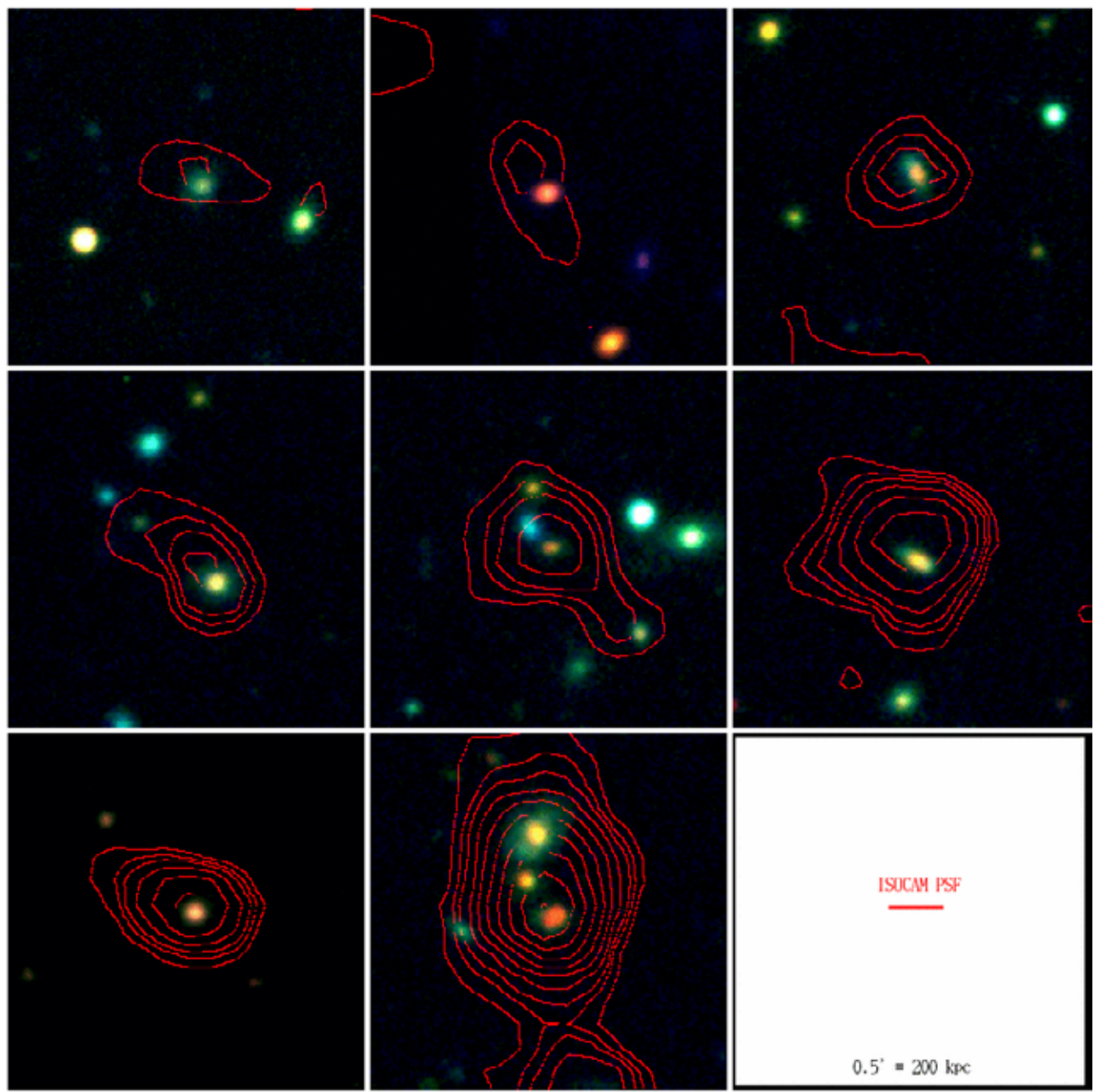

Figure 1. Mid-infrared emitters towards the rich cluster of galaxies J1888.16CL. The $15 \mu \mathrm{m}$ emission is shown with contours corresponding to different signal to noise ratio levels starting with a SNR of 3. In this figure are presented the six ISOCAM emitters firmly identified with galaxies having a measured spectroscopic redshift consistent with cluster membership. The two bright sources at the bottom may lie in groups falling towards the cluster.

\section{LIRGs in the cluster J1888.16CL}

A total of 44 sources were detected at $15 \mu \mathrm{m}$ and the vast majority were unambiguously identified on optical/near-infrared images, thanks to the fair spatial resolution of ISOCAM (4.6" at $15 \mu \mathrm{m}$; see Fig. 1). Among the 27 objects for which spectra could be obtained, six have a redshift consistent with cluster membership while two other sources with slightly higher redshifts may lie in infalling groups. The photometric redshift of all remaining mid-infrared emitters are consistent with a foreground or background location. The most distant source at a redshift of 1.7 is an atypical hyperluminous broad absorption line quasar (Duc et al., 2002). The ISO sources in the cluster are uniformly distributed along the $6 \times 1.3 \mathrm{Mpc}^{2}$ strip mapped by ISOCAM and have a velocity disper- 
sion of $700_{-260}^{+520} \mathrm{~km} \mathrm{~s}^{-1}$, which is marginally comparable to that measured for all cluster members $\left(450_{-80}^{+110} \mathrm{~km} \mathrm{~s}^{-1}\right)$.

The confirmed cluster members detected by ISOCAM have $15 \mu \mathrm{m}$ fluxes ranging between 200 and $750 \mu \mathrm{Jy}$. We estimated their total infrared luminosity using the midinfrared to far-infrared correlation that has been well established for nearby galaxies observed with the IRAS satellite (Chary \& Elbaz 2001). All sources have an inferred IR luminosity above $1.3 \times 10^{11} \mathrm{~L}_{\odot}$, and hence may be considered as genuine Luminous Infrared Galaxies (see Fig. 2). Either nuclear activity or star formation could heat the dust responsible for the infrared emission. Recently, a high number of galaxies shining in the hard X-rays (Martini et al., 2002; Johnson et al., 2003) and in the radio centimetric regime (Morrison \& Owen 2003; Best, 2004) was found in Chandra images and Very Large Array maps of distant clusters, respectively. They are suspected to host an AGN. However, studies based on infrared data obtained with ISO and X-ray data collected by XMM-Newton and Chandra indicate that, statistically, infrared-selected luminous galaxies are predominantly powered by star formation, at least in the field environment (Fadda et al., 2002). All J1888 ISO sources have weak emission lines in their optical spectrum; none of the latter are broad enough to be classified as type 1 AGNs. They typically exhibit an [OII]3727 emission line with a moderate equivalent width (less than $40 \AA$ ) and strong early Balmer Hydrogen absorption lines (the equivalent width of $\mathrm{H} \delta$ is above $4 \AA$ ). This spectroscopic signature is best explained by spectrophotometric models including a selective dust extinction depending on the stellar age and a high rate of current star formation (Poggianti et al., 2001). Such an optical signature gives further support to the hypothesis that the infrared emission detected in the cluster results from a dust enshrouded starburst. From the inferred total infrared luminosities, one may deduce individual star formation rates (SFR) ranging between 20 and $120 \mathrm{M}_{\odot} \mathrm{yr}^{-1}$ (a maximum of $270 \mathrm{M}_{\odot} \mathrm{yr}^{-1}$ including the two ULIRGs for which the cluster membership is not assessed).

\section{Triggering the LIRG/starburst phase in the cluster}

The discovery of extremely active galaxies in $\mathrm{X}$-ray luminous clusters was a priori unexpected given all the processes that eventually inhibit star formation in such an environment. Optical surveys of local clusters indicate a lack of galaxies with blue colors or exhibiting emission lines in their spectrum and hence showing signs of star formation activity. Such galaxies are however more numerous in more distant and younger clusters (Dressler et al., 1999). Another evolutionary effect is the increased number of galaxies with spectra characterized by strong Balmer absorption lines and no emission lines. This signature is consistent with that expected for galaxies having suffered a recent but now faded intense star formation episode (Poggianti et al., 1999). This interpretation however was challenged (Balogh et al., 1999) since prompt truncated star formation may have the same effect in some cases. The presence of post-starburst galaxies implies that starbursts may occur in clusters; they would have remained unnoticed in optical surveys because of their dust cocoon.

What triggered the enhanced infrared activity in the ISO J1888 galaxies? The answer will very much depend on when and where the LIRG event was switched on.

One simple hypothesis is that the mid-infrared emitters have recently been accreted by the cluster and that the luminous infrared phase actually started in the coeval field where LIRGs are not uncommon. Under this assumption, the infall rate that would account for the number of detected galaxies is constrained by the duration of the infrared luminous phase which cannot exceed 100 Myr. During that period at least six field LIRGs should 


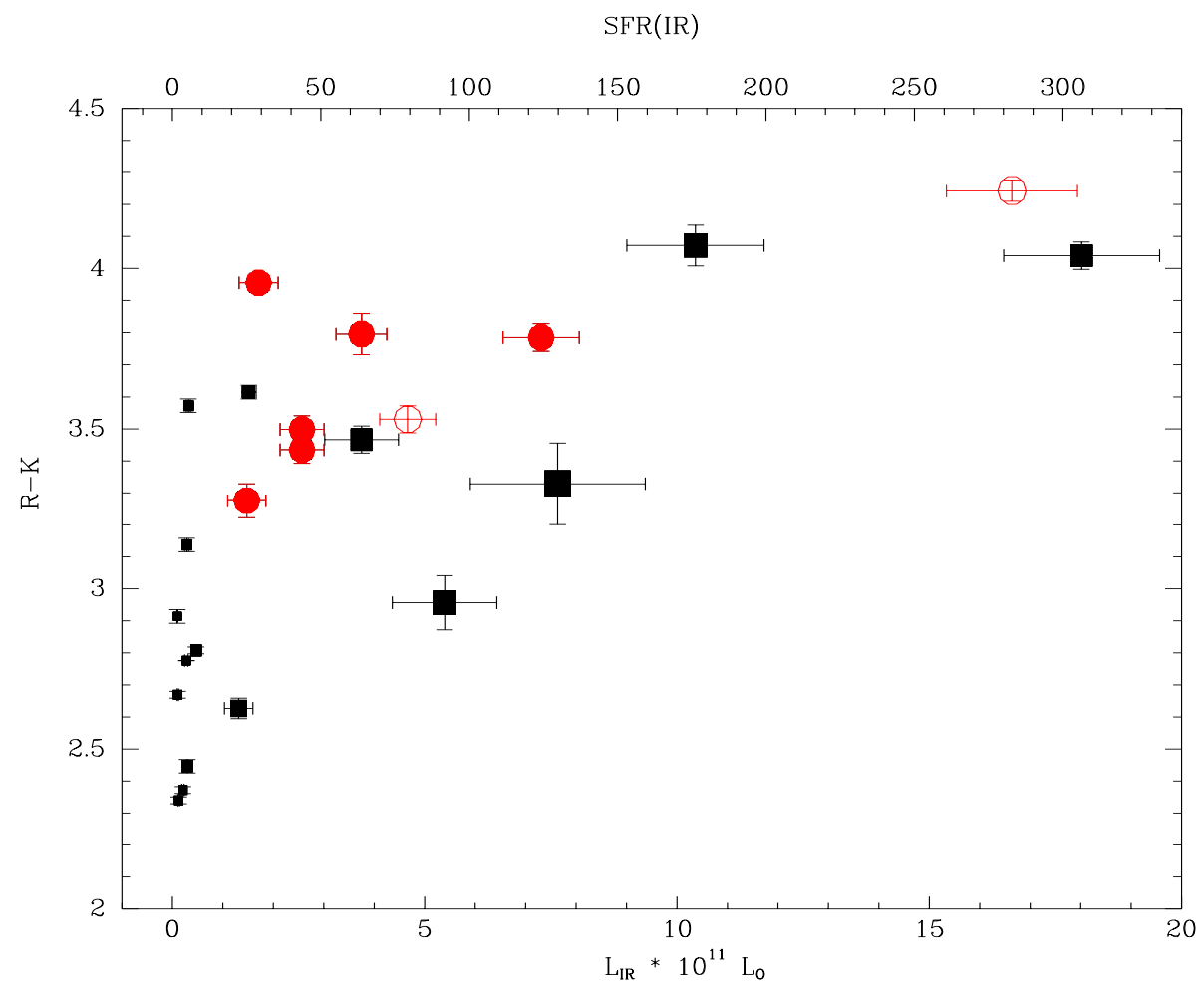

Figure 2. Photometric R-K color index versus the inferred Infrared luminosity of the ISOCAM-detected galaxies towards the field of J1888.16CL. The size of each dot is proportional to the galaxy redshift. The Star Formation Rates in solar masses per year are indicated along the top axis. They were computed from the mid-infrared luminosities. Confirmed cluster members are shown with (red) dots while the two galaxies likely associated with infalling groups are indicated with the two empty circles. The black filled squared are either foreground or background galaxies.

have been accreted. The proportion of LIRGs in the field is still poorly known $\dagger$ but could be of the order of 5-10\% in the redshift interval 0.5-0.6 (Flores et al., 1999). The total inferred infall rate (including the IR-quiet galaxies) would then be of about 100 massive galaxies in $100 \mathrm{Myr}$, which seems unrealistic. Numerical simulations and $\mathrm{X}$-ray observations show however that accretion onto clusters from the field is not a spherically symmetric process, but occurs along filaments or via mergers with other groups and clusters. One therefore cannot exclude the possibility that the LIRGs observed in J1888.16CL belonged to such a recently accreted structure, where tidal interactions could be an efficient mechanism in triggering the star formation.

The alternative is that the luminous infrared phase and associated starburst event occurred within the cluster. There the large velocity dispersion does not favor tidal galaxygalaxy collisions and mergers. Ram-pressure may create some shocks and instabilities in the gaseous component while it is stripped; the changing global cluster tidal field may destabilize the galaxy disks, when they lie in infalling groups (Bekki, 1999) or when a cluster-cluster merger is involved (Miller \& Owen, 2003). Such mechanisms can account for a mild star formation episode but are probably not efficient enough to concentrate

$\dagger$ Whether the field actually exists as such is even questionable. 
large quantities of gas in the nuclear regions, as required to trigger a starburst or an AGN activity with an intensity comparable to the local LIRGs.

\section{Conclusions}

Although the effective triggering mechanism remains unclear -perhaps tidal interactions in sub-structures -, our observations clearly indicate that a significant activity may occur in clusters, even at moderate redshifts and hence a long time after their collapse and the formation of the bulk of their stellar populations. This result is corroborated by other studies based on ISOCAM mid-infrared data (Ricardo-Perez et al., in these proceedings), optical data (see the review by A. Dressler), radio continuum maps and perhaps millimetric SCUBA surveys (Webb et al., these proceedings; Best, 2002). The MIPS camera on board the infrared satellite Sptizer will also very soon provide ample information on the dust enshrouded activity in clusters. This secondary peak of activity may correspond to a phase in the evolution of clusters when accretion of galaxies, especially through the merging of groups, was conspicuous.

\section{References}

Balogh, M. L., Morris, S. L., Yee, H. K. C., et al., 1999, ApJ 527, 54

Bekki, K., 1999, ApJ 510, L15

Best, P. N., 2002, MNRAS 336, 1293

Best, P. N., 2004, MNRAS in press (astro-ph/0402523)

Chary, R., Elbaz, D., 2001, ApJ 556, 562

Dressler, A., Smail, I., Poggianti, B. M., et al., 1999, ApJ 122, 51

Duc, P.-A., Hall, P. B., Fadda, D., et al., 2002, A\&A 389, L47

Fadda, D., Flores, H., Hasinger, G., et al., 2002, A\&A 383, 838

Flores, H., Hammer, F., Thuan, T. X., et al., 1999, ApJ 517, 148

Johnson, O., Best, P. N., Almaini, O., 2003, MNRAS 343, 924

Martini, P., Kelson, D. D., Mulchaey, J. S., Trager, S. C., 2002, ApJ 576, L109

Miller, N. A., Owen, F. N., 2003, AJ 125, 2427

Morrison, G. E., Owen, F. N., 2003, AJ 125, 506

Poggianti, B. M., Smail, I., Dressler, A., et al., 1999, ApJ 518, 576

Poggianti, B. M., Bressan, A., Franceschini, A., 2001, ApJ 550, 195 